\title{
Historia
}

\section{Los manuales de formación de la Cruz Roja durante la epidemia de gripe de 1918-1919}

\section{Spanish Red Cross' training manuals during the 1918-1919 influenza epidemic}

\section{Manuais de formação da Cruz Vermelha Espanhola durante a epidemia de gripe de 1918-1919}

\author{
Alberto González García \\ Doctor en Investigación Sociosanitaria. \\ Facultad de Enfermería de Cuenca, Universidad de Castilla-La Mancha.
}

Cómo citar este artículo en edición digital: González García, A. (2013) Los manuales de formación de la Cruz Roja durante la epidemia de gripe de 1918-1919. Cultura de los Cuidados (Edición digital) 17, 36. Disponible en: <http://dx.doi.org/10.7184/ cuid.2013.36.03>

Correspondencia: Alberto González García: Facultad de Enfermería de Cuenca, Universidad de Castilla-La Mancha. Cuenca. Tlfno.969.17.91.00, extensión 46.33 Correo electrónico: alberto.gonzalez@uclm.es

Recibido 04/03/2013. Aceptado: 14/05/2013.

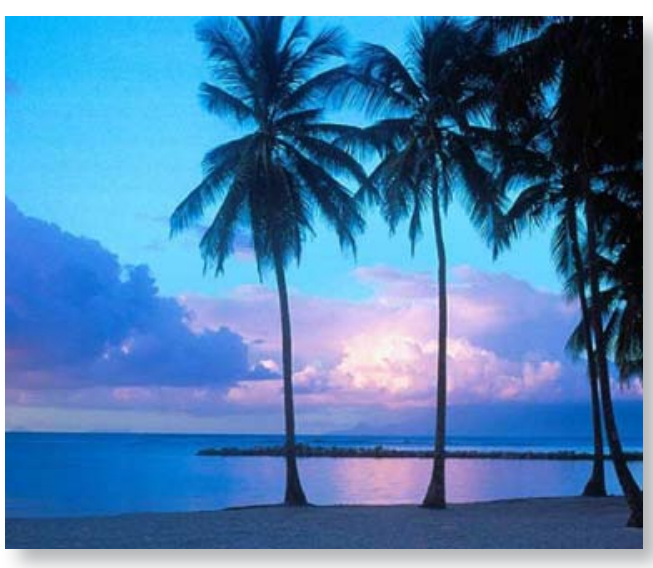

\section{ABSTRACT}

The target of this article is to analyze the training manuals of practitioners, nurses and Spanish Red Cross nurses to assess how far we can place the introduction of educational content related to public health nursing. The reporting period covers from the configuration of the nursing profession to onset of the first figure with real functions of public health nursing, pediatric nurses. We have selected seven training manuals from the Red Cross, three practitioners training manuals, and five nurses training manuals, published until 1925, to compare the contents of each curriculum. The main conclusions are that the Red Cross stands as one of the protagonists of public health nursing in Spain, due to her performance in times of war and, especially, in times of the influenza epidemic of 19181919. Care infectious patients requiring first appeared in the training manuals of the Red Cross nurses following the 1918 influenza epidemic events.

Keywords: Spanish Red Cross, influenza, 1918-1919, training manuals.

\section{RESUMO}

O objetivo é analisar os manuais de formação de practicantes, enfermeiras e enfermeiras da Cruz Vermelha Espanhola 
para avaliar até onde podemos colocar a introdução de conteúdos pedagógicos relacionados à enfermagem de saúde pública. $\mathrm{O}$ período do relatório cobre a partir da configuração da profissão de enfermagem ao início da primeira figura com funções reais de enfermagem de saúde pública, enfermeiras do berçário. Nós selecionamos sete manuais de treinamento enfermeiras da Cruz Vermelha, três manuais de practicantes, e cinco manuais de enfermeiras, publicados até 1925, para comparar o conteúdo de cada currículo. As principais conclusões são que a Cruz Vermelha se destaca como um dos protagonistas da enfermagem de saúde pública em Espanha, em grande parte devido ao seu desempenho em tempos de guerra e, especialmente, em tempos de epidemia de gripe de 1918-1919. Importam pacientes infecciosas que requerem apareceu pela primeira vez os manuais de formação das enfermeiras da Cruz Vermelha após os eventos de 1918 epidêmicas.

\section{Palavras-chave: Cruz Vermelha} Espanhola, gripe, 1918-1919, manuais de formação.

\section{RESUMEN}

El objetivo es analizar los manuales de formación de practicantes, enfermeras y enfermeras de la Cruz Roja para valorar en qué momento podemos situar la introducción de contenidos didácticos relacionados con la enfermería de salud pública. El periodo analizado abarca desde la configuración de la profesión de enfermera hasta la aparición de la primera figura con funciones reales de enfermería de salud pública, las enfermeras puericultoras. Se han seleccionado siete manuales de formación de enfermeras de la Cruz Roja, tres manuales de formación de practicantes, y cinco manuales de formación de enfermeras, editados hasta
1925, para comparar los contenidos de cada plan de estudios. Las principales conclusiones son que la Cruz Roja se sitúa como una de las protagonistas de la enfermería de salud pública en España, debido en gran parte a su actuación en tiempos de guerra y, sobre todo, en tiempos de la epidemia de gripe de 1918-1919. Los cuidados que requieren los enfermos infecciosos aparecieron por primera vez en los manuales de formación de las enfermeras de la Cruz Roja a raíz de los acontecimientos epidémicos de 1918.

Palabras clave: Cruz Roja, gripe, 19181919, manuales de formación.

\section{INTRODUCCIÓN}

En los últimos años XIX y en las primeras décadas del siglo XX nuestra disciplina se encontraba en la búsqueda de su identidad profesional, lo cual permitió que comenzara a dibujarse una conciencia de la praxis que culminaría en la profesionalización de la misma con la formación reglada de los individuos (Herrera Rodríguez, 1996; Feria Lorenzo et al, 2004; Parrilla Saldaña y García González, 2004).

Hasta 1857, la única profesión reconocida relacionada con los cuidados era la de practicante, cuya formación se realizaba en el seno de las Facultades de Medicina. Mediante la Ley de Instrucción Pública de septiembre de 1857, conocida por el nombre del ministro que la impulsó, Claudio Moyano, se dio estatuto legal a la práctica de los cuidados bajo dos denominaciones diferentes, practicante y matrona (Domínguez Alcón, 1986; Camaño Puig y Forero Rincón, 1998; García Martínez y García Martínez, 2004; Ortiz Gómez, 2005).

La Ley Moyano de 1857 regulaba el acceso y estudios de practicante. El plan de estudios 
recogía que los practicantes eran varones que suplían aquellas áreas de la medicina que requerían destrezas manuales, en el aspecto más técnico y pericial de la profesión de enfermería (Domínguez Alcón, 1986; Hernández Martín, Pinar García y Moreno Roy, 1996; Parrilla Saldaña y García González, 2004; Fernández Mérida, 2008). Las mujeres no tenían derecho a acceder a estos estudios hasta 1904. En teoría, estas practicantas estaban igualmente habilitadas para el ejercicio la profesión. Sin embargo en la práctica, las practicantas cubrían la atención de las enfermas de cirugía a su cargo, mantenían, esterilizaban y preparaban los aparatos y material quirúrgico, pasaban visita con los facultativos y anotaban los medicamentos que requería cada paciente. Administraban medicinas, observaban los síntomas para informar al médico en caso de complicación, realizaban curas y en las salas de partos (González Canalejo, 2005). Al contrario de lo que ocurrió con los practicantes, en donde su actividad asistencial estuvo limitada a los cuidados más técnicos, las primeras practicantas contemporáneas desempeñaron un papel decisivo en la comunicación con los enfermos y en la educación sanitaria, puesto que también ejercieron la asistencia domiciliaria (González Canalejo, 2007).

Será a partir de 1915 cuando encontremos el intento de poner en marcha una profesión exclusivamente femenina denominada enfermería. El título de enfermera se reconoció como una profesión distinta a la de los practicantes y se estableció el primer programa oficial para su formación según las directrices de la Ley de Instrucción Pública de 1904 (Domínguez Alcón, 1986; Herrera Rodríguez, 1996; Santo Tomás Pérez, 1997; Parrilla Saldaña y García González, 2004; Ortiz Gómez, 2005). La formación práctica tenía lugar en los hos-

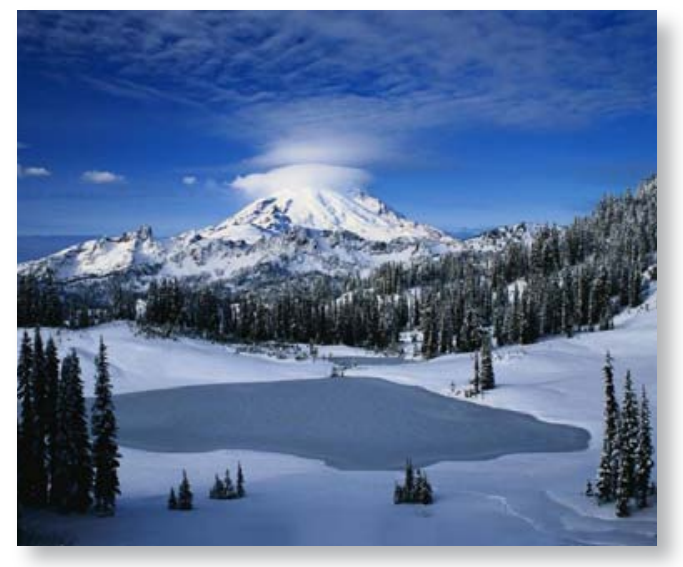

pitales, fueran religiosos o no, y las Facultades de Medicina eran las encargadas de acreditar el nivel de conocimientos de las aspirantes, pero no tenían por qué proveer la formación (Sánchez González, Ortega Martínez y Elbal Moreno, 1996; Ortiz Gómez, 2005).

En 1923 las diferencias entre un practicante y una enfermera eran evidentes. El practicante era considerado una persona perita, con un título profesional de esfera limitada pero respetable como cualquier otra carrera oficial, que ejecutaba prescripciones del médico con arreglo a la ciencia. Sin embargo, la enfermera era cualquier persona que hacía lo que el médico ordenaba con arreglo a la práctica (Hernández Martín, Pinar García y Moreno Roy, 1996). La orientación de estas dos categorías profesionales fue diferente, y también lo fue su formación (cf. Domínguez Alcón, 1986). En este sentido, nos centraremos en la formación de practicantes y enfermeras para abordar este aspecto de forma específica.

Los orígenes de la enfermería comunitaria en Europa se sitúan en Gran Bretaña y se remontan a mediados del siglo XIX, desarrollada en Liverpool durante 1859 y promovida por el cuáquero y filántropo William Rathbone, quien contactó con Florence Nightingale para solicitar información acerca de cómo lle- 
var adelante su proyecto de formación de enfermeras comunitarias (Siles González, 1999; Bostridge, 2008). En nuestro país, el desarrollo de la enfermería de salud pública vendría de la mano de las ideas de Concepción Arenal, quien publicó en varios volúmenes su exhaustivo análisis de la realidad sociosanitaria visitadora española. El retraso en el tiempo del desarrollo de la enfermería comunitaria en nuestro país en relación con los países de nuestro entorno ha sido atribuido, en primer lugar, al patrón socioeconómico español y a la inexistencia de un movimiento sanitario como el inglés. También, a que el asentamiento de la enfermería comunitaria precisa de un clima democrático para poder desarrollarse con normalidad (Siles González, 1999). Además, en España ha existido siempre una larga tradición profesional de la enfermería en el ámbito hospitalario, materializada con las figuras de los practicantes, en un primer momento, y con las enfermeras, posteriormente. Este hecho condicionaría la idea de que la asistencia extrahospitalaria no fuera entendida como una función ni de los practicantes ni de las enfermeras (Galiana Sánchez, Bernabeu Mestre y García Paramio, 2010).

El principal referente tangible en el origen de la profesionalización de la enfermería de salud pública se encuentra en la Instrucción General de Salud Pública de 1904 (Gaceta de Madrid, 1904a; 1904b). Esta norma permitió la creación de las Juntas Provinciales de Sanidad y estableció la conveniencia de que cada Junta procurara organizar una comisión de señoras para la vigilancia de la asistencia domiciliaria a enfermos pobres, propaganda de la higiene durante la lactancia, higiene de párvulos y protección de embarazadas y puérperas pobres. Estas tareas, ligadas a los cuidados maternoinfantiles y a la asistencia domiciliaria, pueden considerarse el precedente de la labor de la enfermería comunitaria (Domínguez Alcón, 1986; Siles González, 1999; Galiana Sánchez, Bernabeu Mestre y García Paramio, 2010).

Sin embargo, a pesar de que la legislación para practicantes y matronas fue relativamente abundante durante este periodo, no se legisló nada respecto a la formación o enseñanzas de las señoras que debían integrar estas comisiones, y cuando en 1915 la ley estableció la titulación de enfermera, la orientación de los estudios y de las actividades profesionales tampoco contemplaron este aspecto (Fernández Mérida, 1998; Parrilla Saldaña y García González, 2004; Galiana Sánchez, Bernabeu Mestre y García Paramio, 2010). Únicamente encontramos actividad de enfermería de salud pública en el colectivo de las primeras practicantas contemporáneas (González Canalejo, 2007). Habrá que esperar veinte años más para retomar formalmente este campo de actuación, cuando en 1925 aparezcan las enfermeras puericultoras, o en 1932 las enfermeras visitadoras, en donde se normativizan las funciones de supervisión y educación sanitaria de las familias y de las amas de casa (Bernabeu Mestre y Gascón Pérez, 1999; Santo Tomás Pérez, 1997; Ortiz Gómez, 2005).

Merece especial atención la Cruz Roja Española, ya que a lo largo de las primeras décadas del siglo XX, la labor llevada a cabo por esta institución y la elaboración de planes de estudio situó a esta institución como protagonista dentro del capítulo de la historia moderna de la enfermería española. La Cruz Roja tuvo una destacada labor de formación de personal sanitario, y en 1920 creó su primera escuela de enfermeras en Madrid, meses después en Barcelona, y en 1927 el Dispensario Central de Madrid (Camaño Puig y Forero Rincón, 1998; Expósito González, 2010). 


\section{OBJETIVOS}

El objetivo de este artículo es releer y analizar los manuales de formación de practicantes, enfermeras y enfermeras de la Cruz Roja editados hasta 1925 para valorar en qué modo y en qué momento podemos situar la introducción de contenidos didácticos relacionados con la enfermería de salud pública. Este periodo abarca tanto la configuración de la profesión de enfermera como la aparición de la primera figura con funciones reales de enfermería de salud pública, las enfermeras puericultoras.

Nuestra hipótesis de partida es la creencia de que la enfermería de salud pública española alcanzó un importante grado de institucionalización con la epidemia de gripe de 1918-1919, lo cual quedó reflejado en los programas de formación de enfermeras de la Cruz Roja de 1917 y, posteriormente, en las enfermeras visitadoras de la Cruz Roja de 1923, al tiempo que se conformó un nuevo espacio alejado de los espacios profesionales de los practicantes en el que concentrar a estas mujeres.

\section{MATERIAL Y MÉTODOS}

Se ha seleccionado una muestra de siete manuales de formación de enfermeras de la Cruz Roja. Además, se han consultado tres manuales de formación de practicantes y, finalmente, cinco manuales de formación de enfermeras, todos ellos editados hasta 1925, para comparar los contenidos de cada plan de estudios. En concreto, los manuales analizados se muestran en las tablas siguientes:

Tabla 1: Manuales de formación de la Cruz Roja

\begin{tabular}{|c|l|l|}
\hline Fecha edición & \multicolumn{1}{|c|}{ Título } & \multicolumn{1}{|c|}{ Autor/a } \\
\hline $\mathbf{1 9 1 7}$ & Manual de la enseñanza de enfermeras & $\begin{array}{l}\text { Mariano Fernández-Corredor } \\
\text { Chicote }\end{array}$ \\
\hline $\begin{array}{c}\mathbf{1 9 1 8}\left(1^{\mathrm{a}} \text { ed.) }\right. \\
\mathbf{1 9 2 0}\left(2^{\mathrm{a}} \text { ed.) }\right.\end{array}$ & El consultor de la Dama Enfermera & Elena Sánchez de Arrojo \\
\hline $\mathbf{1 9 1 8}$ & $\begin{array}{l}\text { Manual de las Damas Enfermeras de la } \\
\text { Cruz Roja }\end{array}$ & Andrés Martínez Vargas \\
\hline $\mathbf{1 9 2 0}$ & Curso teórico de la Dama Enfermera & $\begin{array}{l}\text { María González-Neira de } \\
\text { Terrero }\end{array}$ \\
\hline $\mathbf{1 9 2 1}$ & $\begin{array}{l}\text { Vademécum de la Dama Enfermera de la } \\
\text { Cruz Roja y Auxiliares Sanitarios }\end{array}$ & Mª Dolores Bas Bonald \\
\hline $\mathbf{1 9 2 3}$ & $\begin{array}{l}\text { Contestaciones al programa de examen } \\
\text { teórico para obtener el titulo de Dama } \\
\text { Enfermera de segunda clase }\end{array}$ & Junta de Damas de Pamplona \\
\hline $\mathbf{1 9 2 3}$ & $\begin{array}{l}\text { Lecciones para las Damas Enfermeras de } \\
\text { Cruz Roja de Las Palmas de Gran Canaria }\end{array}$ & Cruz Roja de Gran Canaria \\
\hline
\end{tabular}

Tabla 2: Manuales de formación de practicantes

\begin{tabular}{|c|l|l|}
\hline Fecha edición & \multicolumn{1}{|c|}{ Título } & \multicolumn{1}{|c|}{ Autor } \\
\hline & $\begin{array}{l}\text { Vademécum del practicante: tratado de } \\
\text { cirugia menor }\end{array}$ & Juan Marsillach Parera \\
\hline $\mathbf{1 8 7 0}$ & Manual del practicante & Bonifacio Blanco Torres \\
\hline $\mathbf{1 9 1 6}$ & Manual del practicante & J. Vallory \\
\hline
\end{tabular}

Tabla 3: Manuales de formación de enfermeras

\begin{tabular}{|c|l|l|}
\hline Fecha edición & \multicolumn{1}{|c|}{ Título } & \multicolumn{1}{|c|}{ Autor/a } \\
\hline $\mathbf{1 8 9 4}$ & Manual de la Enfermera & Miguel A. Seco \\
\hline $\mathbf{1 9 0 7}$ & $\begin{array}{l}\text { Manual práctico de la enfermera } \\
\text { hospitalaria y doméstica }\end{array}$ & J. Vilar \\
\hline $\mathbf{1 9 1 5}$ & Los cuidados de malalts & Teresa Font et al \\
\hline $\mathbf{1 9 1 6}$ & Arte de cuidar a los enfermos & L. Grenet \\
\hline $\mathbf{1 9 1 9}$ & La enfermera moderna & Baltasar Pijoán \\
\hline
\end{tabular}




\section{DISCUSIÓN Y CONCLUSIONES}

La reina Isabel II fundó la Cruz Roja por Real Orden de 1864, de acuerdo con las bases establecidas en la Conferencia de Ginebra de octubre de 1863, inspirada en la labor realizada por Florence Nightingale durante la guerra de Crimea (Siles González, 1995; Expósito González, 2010). Los estatutos de la Cruz Roja Española se aprobaron en 1868 y, desde ese momento, la Cruz Roja estaría presente en la epidemia de cólera de 1885, en las inundaciones de Gijón en 1903, en la Semana Trágica de Barcelona de 1909, en la epidemia de tifus de Gijón en 1911, en la Huelga General de 1917, o en la epidemia de gripe de 1918-1919 (Chamizo Vega, 2009; Expósito González, 2010).

El Real Decreto de 28 de febrero de 1917, impulsado por la reina Victoria Eugenia de Battenberg, dictó las instrucciones para la organización del Cuerpo de Damas Enfermeras de la Cruz Roja en nuestro país (Siles González y García Hernández, 1995; Herrera Rodríguez, 1996; Chamizo Vega, 2009; Expósito González, 2010). La obtención del título de Dama Enfermera exigía la aprobación de un examen reglamentario ante un tribunal, así como superar las prácticas reglamentarias realizadas en los hospitales correspondientes (Herrera Rodríguez, 1996; Siles González, 2011).

En el caso que nos ocupa, creemos que existe una vinculación entre estas enfermeras y el campo de la salud pública, cuando en la conformación del cuerpo de Damas Enfermeras de la Cruz Roja se explicitaba la idea de que las mujeres burguesas de la alta sociedad española se involucrasen desinteresadamente en iniciativas de carácter preventivo (Siles González y García Hernández, 1995; Herrera Rodríguez, 1996; Chamizo Vega, 2009; Expósito González, 2010), en un momento en el que la que la higiene pública se empezaba a consti- tuir como un campo científico de la medicina (Rodríguez Ocaña, 2005).

Con motivo de la promulgación del Real Decreto de febrero de 1917, en donde se aprobaba el programa de las enseñanzas, se publicaron los primeros textos didácticos. El programa contaba con treinta y cinco lecciones. Las Damas Enfermeras realizaban dos cursos de formación en periodos de cinco meses por año y realizaban sus prácticas en dispensarios, sin estar sometidas al internado (Expósito González, 2010). Resulta de interés centrarse en la formación de las Damas Enfermeras para valorar, por un lado, cómo se introdujeron conceptos de salud pública relacionados con la higiene y prevención de grandes epidemias a partir de la irrupción de la epidemia de gripe en nuestro país.

Todos los manuales de formación de enfermeras de la Cruz Roja que hemos consultado se caracterizaron por reproducir fielmente las directrices del reglamento de enseñanzas, enfocadas a la clínica y al aprendizaje de técnicas, con una introducción a la historia y organización de la institución, la organización de campañas, así como una referencia a la puericultura y la salud materno-infantil (Chamizo Vega, 2009). Por otro lado, las últimas lecciones de los libros se centraban en la acción de la Cruz Roja en tiempos de paz, detallando aquellas dirigidas a la protección de la infancia, la tuberculosis, la salubridad pública, la higiene social y las enfermedades contagiosas (Galindo Casero et al, 2004).

De igual modo, en todos estos manuales se exponían las condiciones morales, físicas e intelectuales que se les exigía a las mujeres, destacando la discreción, la instrucción, la observación, la subordinación, la fortaleza, la paciencia, la puntualidad, y el secreto profesional (Herrera Rodríguez, 1996). Bajo esta perspec- 
tiva, el Manual de las Damas Enfermeras de la Cruz Roja publicado por el primer director de la Escuela de la Cruz Roja de Barcelona, el doctor Andrés Martínez Vargas, se comparaba la enfermera ideal con la Hermana de la Caridad (Martínez Vargas, 1917; Siles González y García Hernández, 1995).

La Dama Enfermera era la encargada de proporcionar cuidados maternales a los heridos de guerra, necesitados de dulzura y ternuras de mujer, siempre en términos de corrección, consuelo y caridad cristianos (Sánchez de Arrojo, 1920). Además, estaba subordinada a las disposiciones de los médicos, sin pretender jamás sustituirle (Sánchez de Arrojo, 1920; Herrera Rodríguez, 1996). Pese a lo reducido del temario comparado con el temario de la formación de enfermeras, a través de este análisis observamos cómo se repiten los mismos valores de filantropía, cuidado maternal y religioso atribuidos a la figura de la enfermera (Keller, 1991; Miqueo Miqueo, 2001; Ortiz Gómez, 2001; Esteban Galarza, 2004).

En 1918 encontramos El consultor de la Dama Enfermera, publicado en Guadalajara por una integrante del Cuerpo de Damas Enfermeras, Elena Sánchez de Arrojo (Expósito González, 2010). En 1920 se publicó la segunda edición (Imagen 1).

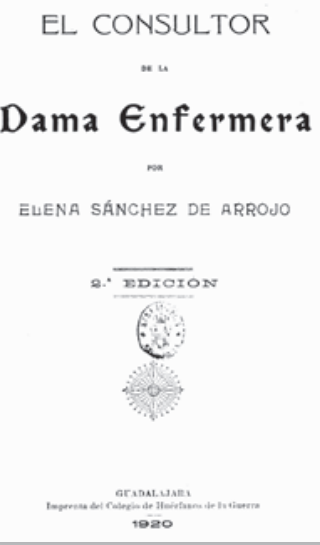

Imagen 1: Portada del manual El consultor de la Dama Enfermera
Elena Sánchez era una mujer viuda de la burguesía alcarreña, que desde su militancia católica tenía inquietudes por las obras sociales. En este sentido, se dedicó al ejercicio de la caridad como Dama de la Junta del Hospital de la Princesa (Calatraveño, 1920). Sus ideas sociales y religiosas llevaron a esta mujer a seguir el curso que para las Damas Enfermeras dictaron los médicos Joaquín García Plaza y Eduardo del Río, realizando el examen ante el primer tribunal que se constituyó. Estamos, pues, ante una de las pioneras de este cuerpo de Damas enfermeras, no sólo en Guadalajara, sino también a nivel nacional (Herrera Rodríguez, 1996; Expósito González, 2010).

La segunda edición del manual de Elena Sánchez de Arrojo introdujo por primera vez conceptos como la infección, el estudio de los principales agentes microbianos, las complicaciones infecciosas de las heridas, las principales epidemias, los conceptos de asepsia y antisepsia, y el uso de los principales desinfectantes, así como consejos concretos sobre especial atención en tiempos de epidemia y durante la visita médica (Sánchez de Arrojo, 1920; Herrera Rodríguez, 1996):

“(...) esta segunda edición corregida y aumentada con materias que aunque no figuran en el programa oficial, me han parecido de suma utilidad. Tales son las que se refieren á la profilaxis contra la grippe, erisipela y sarampión, modo de hacer y aplicar tópicos, sinapismos, vegigatorios, ventosas, etc., de vestir y desnudar á los heridos y mutilados y reeducación de éstos" (Sánchez de Arrojo, 1920: VIII).

La Cruz Roja, por tanto, se sitúa como una de los artífices y protagonistas de la enfermería de salud pública en España, debido en gran parte a su actuación en tiempos de guerra $y$, sobre todo, en tiempos de la epidemia de gripe de 1918-1919. 
Con respecto a la formación de los practicantes, el primer manual que se editó para la formación de practicantes tras la publicación de las enseñanzas fue el Vademécum del practicante: Tratado de cirugía menor, del doctor Juan Marsillach Parera. Este manual fue el más completo de todas las enseñanzas de practicante, aunque en esta época existieron casi treinta manuales. Todos los manuales de formación de practicantes consultados, aunque también los de épocas posteriores según las fuentes secundarias (hasta su remodelación en 1955) presentaron una orientación hacia técnicas concretas de cirugía menor, curas y vendajes, incluyendo como únicos aspectos de la salud pública conceptos de asepsia y antisepsia de la infección aplicados a la cirugía menor, así como la técnica de la vacunación (Domínguez Alcón, 1986; Expósito González, 2004; Antón Solanas, Hallett y Wakefield, 2005).

Por otro lado, se observa en los manuales de formación de enfermeras que desde el primer manual publicado en 1894 se advierte expresamente que la intención del manual era contribuir a vulgarizar el método antiséptico. Hasta la publicación del último manual en 1919, todos los volúmenes dedicados a la formación de enfermeras incluyeron contenidos de asepsia, desinfección, esterilización, higiene y prevención de enfermedades (Domínguez Alcón, 1986).

Como puede comprobarse, el practicante tenía una misión más autónoma y extrahospitalaria, mientras que la enfermera dedicaba su actividad tanto al campo hospitalario como a la asistencia a domicilio (Domínguez Alcón, 1986). La orientación de la asistencia de los practicantes, por tanto, fue diferente a la de la enfermera, puesto que no estaba controlada por la institución médica de la misma forma que la enfermería, ni tuvo una orientación ha- cia la asistencia hospitalaria, sino que su actividad se asemejaba más a aquellas profesiones denominadas liberales o al trabajo por cuenta propia (Ortiz Gómez, 2006). Siguiendo este concepto de asistencia sanitaria, los manuales de los practicantes no incluyeron conceptos de epidemia ni infecciones, sino que sólo incluían formación en materia de vacunación como único contenido relacionado con la salud pública y la higiene (García Martínez, 2004).

Sin embargo, vemos cómo la higiene del paciente, la observación del enfermo en su entorno domiciliario, y los cuidados que requieren los enfermos infecciosos sí están presentes en los manuales de enfermeras. Específicamente, estos contenidos aparecieron por primera vez en los manuales de formación de las enfermeras de la Cruz Roja a raíz de los acontecimientos epidémicos de 1918, momento en el que por primera vez se aplicaron medidas de prevención y aislamiento de enfermos. Se sucederán otros ejemplos posteriores como, en el reglamento para las hermanas enfermeras de los Hospitales militares de 1922, en donde se recogían contenidos relacionados con la evolución de las enfermedades infecto-contagiosas, la infección, el aislamiento, los desinfectantes y los mecanismos de desinfección (Chamizo Vega, 2009).

\section{BIBLIOGRAFÍA}

- Antón Solanas, I.; Hallett, C.E.; Wakefield, A. (2005) La influencia de la Guerra Civil sobre el desarrollo de la profesión de enfermería en España: su evolución a través de un siglo de historia. Temperamentvm 2. Available http://www.index-f.com/temperamentum/tn2/t0124. php

- Bernabeu Mestre, J.; Gascón Pérez, E. (1999) Historia de la enfermería de salud pública en España (1860-1977). Alicante, Universitat d'Alacant. 
- Bostridge, M. (2008) Florence Nightingale: the woman and her legend. Londres, Book Jacket.

- Calatraveño, F. (1920) Prólogo. En: Sánchez de Arrojo, E. El consultor de la Dama Enfermera, Guadalajara, Imprenta del Colegio de Huérfanos de la Guerra: IX-XV.

- Camaño Puig, R.; Forero Rincón, O. (1998) Escuelas de Enfermería: de la escasez a la abundancia. Su evolución histórica (1857-1977). Híades 5-6: 155-167.

- Chamizo Vega, C. (2009) El proceso de profesionalización de la Enfermería en el Principado de Asturias. Alicante, Universitat d'Alacant, 2009.

- Domínguez Alcón C. (1986) Los cuidados y la profesión enfermera en España. Madrid, Pirámide.

- Esteban Galarza, M.L. (2004) Antropología del cuerpo. Géneros, itinerarios corporales, identidad y cambio. Barcelona, Bellaterra.

- Expósito González, R. (2004) Carrera de Practicante (1916). Híades 9: 97-106.

- Expósito González, R. (2010) Los manuales para la formación de las enfermeras de la Cruz Roja espańola. En: González Canalejo, C.; Martínez López, F. eds. La transformación de la Enfermería. Nuevas miradas para la Historia, Granada, Editorial Comares: 311-324.

- Feria Lorenzo, D.J.; León López, R.; Macías Rodríguez, A.C. et al. (2004) Evolución histórica de los cuidados desde el siglo XIX hasta nuestros días. Híades 9: 425-451.

- Fernández Mérida, M.C. (1998). La asistencia domiciliaria y la legislación en el siglo XIX. Híades 5-6: 145-154.

- Fernández Mérida, M.C. (2008) La función del personal de Enfermería en el Hospital Provincial de Málaga en el siglo XIX según el Reglamento de 1861. Híades 10: 583-590.

- Gaceta de Madrid (1904a) Instrucción General de Sanidad, nº 22, 22 de enero, 273-275.

- Gaceta de Madrid (1904b) Instrucción General de Sanidad (continuación), n² 23, 23 de enero, 290-295.

- Galiana Sánchez, M.E.; Bernabeu Mestre, J.; García Paramio, M.P. (2010) Enfermeras para una Nueva Patria. Género e ideología en la Enfermería de Falange. En: González Canalejo, C.; Martínez López, F. eds. La transformación de la Enfermería. Nuevas miradas para la Historia, Granada, Editorial Comares: 203-216.
- Galindo Casero, A. Garvi García, M.; Villa Andrada, J.M.; García Retamar, S. (2004) Curso Teórico de la Dama Enfermera (1920). Híades 9: 89-96.

- García Martínez, M.J. (2004) Instrucción del Practicante ó Resumen de conocimientos útiles para la buena asistencia inmediata de los enfermos; y compendio de las operaciones de cirugía menor, arte del dentista y del callista (1870). Híades 9: 77-87.

- García Martínez, M.J.; García Martínez, A.C. (2004) Mujer y asociacionismo profesional. El Colegio de Matronas de Sevilla y su labor en la defensa del colectivo durante el primer tercio del siglo XX. Híades 9: 607-624.

- González Canalejo, C. (2005) El acceso a la educación universitaria de las primeras practicantas (1900-1931). En: Flecha García, C.; Núñez Gil, M.; Rebollo Espinosa, M.J. dir. Mujeres y educación. Saberes, prácticas y discursos en la historia, Sevilla, Diputación Provincial de Sevilla: 119-133.

- González Canalejo, C. (2007) Cuidados y bienestar: el trabajo sanitario femenino en respuesta a la "cuestión social". Dynamis 27: 211-235.

- Hernández Martín, F.J.; Pinar García, M.E.; Moreno Roy, M.A. (1996) Memoria de un centenario. Primera Escuela de Enfermería Santa Isabel de Hungría. Híades 3-4: 189-195.

- Herrera Rodríguez, F. (1996) La enseñanza de las Damas Enfermeras de la Cruz Roja (1917-1920). Híades 3-4: 197-209.

- Keller, E.F. (1991) Reflexiones sobre género y ciencia. Valencia, Edicions Alfons El Magnànim-Institució Valenciana d’estudis i investigació.

- Martínez Vargas, A. (1917) Manual de las Damas Enfermeras de la Cruz Roja. Barcelona, Jaume Vives.

- Miqueo Miqueo, C. (2001) Semiología del androcentrismo. Teorías sobre reproducción de Andrés Piquer y François Broussais. En: Miqueo Miqueo, C.; Tomás, C.; Tejero, C.; Barral, M.J.; Fernández, T.; Yago, T. eds. Perspectivas de género en salud. Fundamentos científicos y socioprofesionales de diferencias sexuales no previstas, Madrid, Minerva Ediciones: 97-134.

- Ortiz Gómez, T. (2001) El género, organizador de profesiones sanitarias. Miqueo Miqueo, C.; Tomás, C.; Tejero, 
C.; Barral, M.J.; Fernández, T.; Yago, T. eds. Perspectivas de género en salud. Fundamentos científicos y socioprofesionales de diferencias sexuales no previstas, Madrid, Minerva Ediciones: 53-75.

- Ortiz Gómez, T. (2005) Profesiones sanitarias. En: Morant Deusa I. (dir.); Gómez-Ferrer Morant, G.; Barrancos, D.; Lavrin, A. coords. Historia de las mujeres en España y América Latina. Volumen III: Del siglo XIX a los umbrales del XX, Madrid, Cátedra: 523-543.

- Ortiz Gómez, T. (2006) Las mujeres en las profesiones sanitarias (1800-1975). En: Morant Deusa, I. dir. Historia de las mujeres en España y América Latina, Madrid, Cátedra: 489-507.

- Parrilla Saldaña, J.; García González, C. (2004) Análisis de la Enfermería en España desde finales del siglo XIX hasta su integración en la Universidad. Híades 9: 407424.

- Rodríguez Ocaña, E. (2005) Salud Pública en España. Ciencia, profesión y política, siglos XVIII-XX. Granada, Universidad de Granada.

- Sánchez de Arrojo, E. (1920) El consultor de la Dama Enfermera. Guadalajara, Imprenta del Colegio de Huér- fanos de la Guerra.

- Sánchez González, N.; Ortega Martínez, C.; Elbal Moreno, L. (1996) Historia de la Enfermería a través de las instituciones de Castilla-La Mancha. Albacete, Colegio Oficial de Enfermería de Albacete.

- Santo Tomás Pérez, M. (1997) Historia de la Enfermería. En: Fernández Ferrín C.; Garrido Abejar, M.; Santo Tomás Pérez, M.; Serrano Parra, M.D.; Fuentes Chacón, R.M. Máster de Enfermería. Volumen I: Enfermería Fundamental, Barcelona, Masson: 3-131.

- Siles González, J. (1999) Historia de la Enfermería Comunitaria en España. Un enfoque social, político, científico e ideológico de la evolución de los cuidados comunitarios. Index de Enfermería 24-25: 25-31 Available http://www.index-f.com/index-enfermeria/24-25revista/24-25_articulo_25-31.php

- Siles González, J. (2011) Historia de la Enfermería. Madrid, Difusión Avances de Enfermería.

- Siles González y García Hernández (1995) Estudio documental sobre la influencia de la Cruz Roja en la enfermería española (desde sus orígenes hasta la II República). Enfermería Clínica 5 (5): 7-13. 